\title{
Note on Irreducible Diagonally Dominant Matrices and the Convergence of the AOR Iterative Method
}

\author{
By M. Madalena Martins*
}

\begin{abstract}
Considering the linear systems $A x=b$, where the matrix $A$ is irreducible and diagonally dominant, we obtain bounds for the spectral radius of the $L_{r, \omega}$ matrix of the AOR method and we achieve the convergence conditions given in [2] by a different method.

If $\boldsymbol{A}$ is strictly diagonally dominant, we get larger intervals for the parameter $\omega$ of the SOR method, and we improve the results of Theorems 5, 6 of [3] for the AOR method.
\end{abstract}

1. Introduction. The Accelerated Overrelaxation (AOR) Iterative Method for linear systems was presented by Hadjidimos in [2], 1978.

In [3], 1980, we obtained bounds for the spectral radius of the AOR associated matrix and we improved the convergence intervals, given by Hadjidimos, when the matrix of the system is strictly diagonally dominant.

Here we consider irreducible, diagonally dominant matrices, and the results of [3] are extended to these. We get larger convergence intervals for the Successive Overrelaxation Iterative Method (SOR).

In [5] the convergence interval was only $0<\omega<1$; here it is $0<\omega<$ $2 /\left(1+\max _{i}\left(e_{i}+f_{i}\right)\right)$. Our Theorem 6 improves the results of Theorems 4, 5 of [3].

Given the linear system $A x=b$, we consider the following splitting of the matrix $A$ :

$$
A=I-E-F \text {. }
$$

Here $I$ is the identity $(n, n)$ matrix, and $E$ and $F$ are, respectively, $(n, n)$ strictly lower and upper triangular matrices.

We remember that the AOR method of [2] can be written as follows:

$$
\begin{aligned}
x^{(i+1)}= & (I-r E)^{-1}[(1-\omega) I+(\omega-r) E+\omega F] x^{(i)} \\
& +\omega(I-r E)^{-1} b, \quad i=0,1,2, \ldots,
\end{aligned}
$$

with the associated matrix $L_{r, \omega}$ given by

$$
L_{r, \omega}=(I-r E)^{-1}[(1-\omega) I+(\omega-r) E+\omega F] .
$$

The AOR method is the Jacobi extrapolated method if $r=0$, and it is the SOR extrapolated method if $r \neq 0$ with the parameter $\omega / r$. See [3].

\section{Irreducible, Diagonally Dominant Matrices.}

2.1. Bounds for $\rho\left(L_{r, \omega}\right)$. We denote by $\rho\left(L_{r, \omega}\right)$ the spectral radius of the $L_{r, \omega}$ matrix.

Received July 16, 1980; revised November 19, 1980.

1980 Mathematics Subject Classification. Primary 65F10.

- Supported by Instituto Nacional de Investigacão Cientifica, Department of Mathematics, University of Coimbra. 
$A$ is said to be irreducible and diagonally dominant if it is irreducible and satisfies

$$
\left|a_{i i}\right|>\sum_{\substack{j=1 \\ j \neq i}}^{n}\left|a_{i j}\right|, \quad i=1, \ldots, n,
$$

with strict inequality for at least one $i$. Such matrices are nonsingular. See [4].

LEMMA 1. If $A$ is irreducible, then the matrix

$$
P=I-\frac{r(\lambda-1)+\omega}{\lambda-1+\omega} E-\frac{\omega}{\lambda-1+\omega} F
$$

is also irreducible, provided $|\lambda| \geqslant 1,0<\omega<2$, and $\omega>r>0$.

Proof. Under these conditions, the elements of $\boldsymbol{P}$ are different from zero for $|\lambda| \geqslant 1$, and $P$ is irreducible.

THEOREM 1. If $A$ of (1.1) is irreducible and diagonally dominant, then $\rho\left(L_{r, \omega}\right)$ satisfies

$$
\min _{i} \frac{|1-\omega|-|\omega-r| e_{i}-|\omega| f_{i}}{1+r\left|e_{i}\right|}<\rho\left(L_{r, \omega}\right)<\max _{i} \frac{|\omega-r| e_{i}+|\omega| f_{i}+|1-\omega|}{1-|r| e_{i}}
$$

if $|r|<1 / e_{i}$. Here $e_{i}$ and $f_{i}$ are, respectively, the sum of the absolute values of the elements of the ith row of $E$ and $F$, respectively.

Proof. We give a short proof as we follow Theorem 1 of [3]. We see that the eigenvalues of $L_{r, \omega}$ are the roots of det $P=0$, with $P$ given in Lemma 1 .

With $|\lambda| \geqslant 1$, we know that $P$ is irreducible.

We can choose the parameters $\omega$ and $r$ such that $P$ be a diagonally dominant matrix. With these conditions, the values of $\lambda$ such that $|\lambda|>1$ cannot be eigenvalues of $L_{r, \omega}$, because $P$ is singular. Following [3] we obtain the results of the theorem.

We note that $\omega$ and $r$ in Theorem 1 of [3] could take any value, and here $0<\omega<2, \omega>r>0$.

2.2. Convergence conditions for $A O R$ method.

THEOREM 2. If $A$ of (1.1) is irreducible and diagonally dominant with $\omega>r>0$, the AOR method is convergent if

$$
0<\omega<\frac{2}{1+\max _{i}\left(e_{i}+f_{i}\right)} .
$$

Proof. From (2.2), we see that $\rho\left(L_{r, \omega}\right)<1$ if we have

$$
\frac{|\omega-r| e_{i}+|\omega| f_{i}+|1-\omega|}{1-|r| e_{i}}<1,
$$

with strict inequality for at least one $i$.

Following [3], we see that the AOR method will be convergent.

COROLlary 1. If $A$ is irreducible and diagonally dominant, the AOR method is convergent if $0<\omega<1$ and $0<r<1$. 
Proof. Here we have $\max _{i}\left(e_{i}+f_{i}\right)=1$, so $0<\omega<1$. As the AOR method is the extrapolated Jacobi method for $r=0$, we have, from the Extrapolation Theorem given in [3], that $\rho\left(L_{0, \omega}\right)<1$ if $0<\omega<2 /\left(1+\rho\left(L_{0,1}\right)\right)$.

We note that we achieve the Hadjidimos results of [2], by a different method.

\section{Strictly Diagonally Dominant Matrices.}

THEOREM 3. If A of (1.1) is a strictly diagonally dominant matrix, the SOR method converges if

$$
0<\omega<\frac{2}{1+\max _{i}\left(e_{i}+f_{i}\right)} .
$$

Proof. It is evident if we apply Theorem 2 of [3], with $\omega=r$. So, we achieve a larger interval of convergence for the SOR method. It had been proved only for $0<\omega<1,[5]$.

THEOREM 4. If $A$ of $(1.1)$ is strictly diagonally dominant, then $\rho\left(L_{r, \omega}\right)<1$ if

$$
0<r<\frac{2}{1+\max _{i}\left(e_{i}+f_{i}\right)} \text { and } 0<\omega<\frac{2 r}{1+\rho\left(L_{r, r}\right)} \text {. }
$$

Proof. This comes from the Extrapolation Theorem of [3] and from Theorem 3 above.

This result improves the intervals given in [3, Theorem 5].

ThEOREM 5. The AOR method is convergent, $\rho\left(L_{r, \omega}\right)<1$, if

$$
0<r<\frac{2}{1+\max _{i}\left(e_{i}+f_{i}\right)} \text { and } 0<\omega<\frac{2 r}{1+\rho\left(L_{r, r}\right)},
$$

with $2 r /\left(1+\rho\left(L_{r, r}\right)\right) \geqslant 2 /\left(1+\max _{i}\left(e_{i}+f_{i}\right)\right)$;

$$
0<r<\omega \text { and } \frac{2 r}{1+\rho\left(L_{r, r}\right)}<\omega<\frac{2}{1+\max _{i}\left(e_{i}+f_{i}\right)}
$$

or

$$
0<r<\frac{2}{1+\max _{i}\left(e_{i}+f_{i}\right)} \text { and } 0<\omega<\frac{2 r}{1+\rho\left(L_{r, r}\right)},
$$

with $2 r /\left(1+\rho\left(L_{r, r}\right)\right)<2 /\left(1+\max _{i}\left(e_{i}+f_{i}\right)\right)$.

Proof. These results are obtained from Theorem 4 and Theorems 2, 6 of [3].

Here the intervals for the parameters $\omega$ and $r$ are larger than the corresponding intervals of Theorem 6 of [3].

Department of Mathematics

University of Coimbra

Coimbra, Portugal

1. G. Avdelas, A. HAdJmmos \& A. Yeyios, Some Theoretical and Computational Results Concerning the Accelerated Overrelaxation (AOR) Method, Tech. Report No. 8, Department of Mathematics, University of Ioannina, Ioannina, Greece, 1978.

2. A. Hadjidimos, “Accelerated overrelaxation method," Math. Comp., v. 32, 1978, pp. 149-157.

3. M. MARTINS, "On an accelerated overrelaxation iterative method for linear systems with strictly diagonally dominant matrix," Math. Comp., v. 35, 1980, pp. 1269-1273.

4. R. S. VArgA, Matrix Iterative Analysis, Prentice-Hall, Englewood Cliffs, N. J., 1962.

5. D. M. Young, Iterative Solution of Large Linear Systems, Academic Press, New York, 1971. 